\title{
Two visions of clinical integration: perspectives from health system leaders
}

\author{
Wenke Hwang, Joseph Andrie, Michelle LaClair, Harold Paz \\ Penn State University, College of Medicine, Hershey, PA, United States
}

Received: October 10, 2014

Accepted: November 26, 2014 Online Published: December 5, 2014

DOI: $10.5430 /$ jha.v4n1p36

URL: http://dx.doi.org/10.5430/jha.v4n1p36

\begin{abstract}
Objective: Clinical Integration has been implicated as the key to achieving higher quality of care at a lower cost. However, ambiguity regarding the meaning of clinical integration poses challenges as health care professionals strive to adapt to the rapidly evolving health care environment. This study aims to solicit insights from health system executives about what it means to be clinically integrated.

Methods: The authors interviewed 13 health system executives from 11 different institutions in Pennsylvania ranging from small community hospitals to large academic medical centers.

Results: Two major viewpoints of clinical integration were identified from the interviews: patient-centric, which emphasized the importance of the patient's experience and strengthening patient involvement in their own healthcare, and provider-centric, which focused on leadership roles, organizational structure, and physician alignment. Participants with provider-centric viewpoints were associated with larger medical centers and were more likely to describe their health systems as highly clinically integrated. Conversely, patient-centric perspectives were affiliated with smaller health systems/hospitals and were more likely to describe their health systems as less integrated. Participants also identified five key success factors of clinical integration: physician alignment, shared data and analytics, culture, patient engagement, and an emphasis on primary care.

Conclusions: Despite the central role of clinical integration in emerging health systems, there is not a shared understanding of its definition. A better understanding of the varied perspectives regarding clinical integration can help current and future health care professionals to better communicate with one another about clinical integration and the practical steps necessary to achieve it.
\end{abstract}

Key Words: Clinical integration, Health system, Patient-centric, Provider centric, Hospital administration

\section{Introduction}

Recent reform in the U.S. healthcare system has focused on achieving a high performing health care system with improved health, better care, and lower cost. ${ }^{[1]}$ Clinical integration has been heralded as the key to increasing system performance. ${ }^{[2]}$ However, a recent report shows that integrated healthcare systems span a wide spectrum of types and degrees of integration, and the basic definition of clini- cal integration remains unclear. ${ }^{[3]}$ This ambiguity can pose challenges as health care professionals strive to adapt to the rapidly evolving health care environment and healthcare administrators attempt to achieve the triple aim through clinical integration.

Previous research conducted in the mid-1990s called for taxonomy of healthcare systems to provide policymakers with a contextual framework to assess organizational policies and

\footnotetext{
*Correspondence: Wenke Hwang; Email: WHwang@phs.psu.edu; Address: Mail Code A210, 90 Hope Drive, Suite 2200, Hershey, PA 17033,
} United States. 
programs. ${ }^{[4]}$ In 1994, Devers et al. published a "scorecard" to clarify the evolving concept of "clinical integration" of health systems. The authors define clinical integration as "the extent to which patient care services are coordinated across various functions, activities, and operating units of a system $^{[4]}$ ". Six major dimensions to integration are identified: clinical protocol development, medical records uniformity and accessibility, clinical outcomes data collection and utilization, clinical programming and planning efforts, shared clinical support services, and shared clinical service lines. Subsequent updates of this taxonomy have been published; the most recent update in 2004 emphasizes integration of financial structure and physician-system alignment. ${ }^{[5]}$

Since earlier attempts to define clinical integration, the rise in electronic health records (EHR) and call for greater accountability in patient care has dramatically altered how a health system organizes and operates. In addition, policy such as Medicare Hospital Readmission Reduction Program gives new financial meaning to clinical integration. ${ }^{[6]} \mathrm{By}$ interviewing health system executives, a practical, front-line view of clinical integration can be developed. Revisiting the definition of clinical integration is a vital step toward building a highly organized health care system.

\section{Method}

\subsection{Study design}

Qualitative research has routinely been used to examine the complexity of and the interdependencies within a health system. ${ }^{[7]}$ In this study, standardized interviews were utilized to gauge health system executives' perspectives on clinical integration. While individual participants were interviewed for this study, they were not the subject of the study. Their interviews were utilized as expert opinion and, as such, were not determined to be human subject research. Therefore, this study was deemed exempt by the Institutional Review Board of the Penn State College of Medicine.

\subsection{Study sample}

The study sample was identified using snowball sampling. The chain started with one hospital Chief Operating Officer, initially identified through institutional contacts. This initial contact further identified other health system executives for potential interview. Each potential participant was contacted. All participants were asked at the conclusion of each interview to identify additional health system leaders. This resulted in 18 health systems executives being contacted, with 13 agreeing to participate. The titles of the participants included: President, Chief Executive Officer, Chief Operations Officer, Chief Medical Officer, Senior Vice President and Chief Physician Executive. These participants represent a wide spectrum of healthcare organizations including six academic medical centers and five community hospitals.
Key characteristics of the flagship hospital of each health system were abstracted from the American Hospital Association's Data Viewer and are shown in Table 1.

Table 1: Demographics of the flagship hospital associated with each participant

\begin{tabular}{|c|c|c|c|c|}
\hline Interview & $\begin{array}{l}\text { Total } \\
\text { Beds }\end{array}$ & Admissions & $\begin{array}{l}\text { Outpatient } \\
\text { Visits }\end{array}$ & Personnel \\
\hline 1 & $\begin{array}{l}301- \\
500\end{array}$ & $\begin{array}{l}25,001- \\
35,000\end{array}$ & $\begin{array}{l}400,001- \\
600,000\end{array}$ & $6,500+$ \\
\hline 2 & $\begin{array}{l}501- \\
700\end{array}$ & $\begin{array}{l}25,001- \\
35,000\end{array}$ & $\begin{array}{l}800,001- \\
1,000000\end{array}$ & $\begin{array}{l}3,501- \\
4,500\end{array}$ \\
\hline 3 & $700+$ & $\begin{array}{l}35,001- \\
45,000\end{array}$ & $\begin{array}{l}200,000- \\
400,001\end{array}$ & $\begin{array}{l}3,501- \\
4,500\end{array}$ \\
\hline 4 & $\begin{array}{l}501- \\
700\end{array}$ & $\begin{array}{l}25,001- \\
35,000\end{array}$ & $\begin{array}{l}400,001- \\
600,000\end{array}$ & $\begin{array}{l}2,501- \\
3,500\end{array}$ \\
\hline 5 & $\begin{array}{l}501- \\
700\end{array}$ & $\begin{array}{l}25,001- \\
35,000\end{array}$ & $1,000,000+$ & $\begin{array}{l}4,501- \\
5,500\end{array}$ \\
\hline 6 & $700+$ & $\begin{array}{l}35,001- \\
45,000\end{array}$ & $\begin{array}{l}200,000- \\
400,001\end{array}$ & $\begin{array}{l}5,501- \\
6,500\end{array}$ \\
\hline 7 & $\begin{array}{l}100- \\
300\end{array}$ & $\begin{array}{l}5,000- \\
15,000\end{array}$ & $\begin{array}{l}400,001- \\
600,000\end{array}$ & $\begin{array}{l}1,501- \\
2,500\end{array}$ \\
\hline 8 & $\begin{array}{l}301- \\
500\end{array}$ & $\begin{array}{l}25,001- \\
35,000\end{array}$ & $\begin{array}{l}400,001- \\
600,000\end{array}$ & $6,500+$ \\
\hline 9 & $\begin{array}{l}501- \\
700\end{array}$ & $\begin{array}{l}25,001- \\
35,000\end{array}$ & $1,000,000+$ & $\begin{array}{l}4,501- \\
5,500\end{array}$ \\
\hline 10 & $\begin{array}{l}501- \\
700\end{array}$ & $\begin{array}{l}15,001- \\
25,000\end{array}$ & $\begin{array}{l}400,001- \\
600,000\end{array}$ & $\begin{array}{l}2,501- \\
3,500\end{array}$ \\
\hline 11 & $\begin{array}{l}100- \\
300\end{array}$ & $\begin{array}{l}5,000- \\
15,000\end{array}$ & $\begin{array}{l}200,000- \\
400,000\end{array}$ & $\begin{array}{l}500-1, \\
500\end{array}$ \\
\hline 12 & $\begin{array}{l}100- \\
300\end{array}$ & $\begin{array}{l}5,000- \\
15,000\end{array}$ & $\begin{array}{l}200,000- \\
400,000\end{array}$ & $\begin{array}{l}500-1, \\
500\end{array}$ \\
\hline 13 & $700+$ & $45,000+$ & $1,000,000+$ & $6,500+$ \\
\hline
\end{tabular}

\subsection{Survey instrument}

An 11-question interview guide was developed and reviewed by two independent qualitative researchers to ensure clarity and quality of questions. The questions were openended and designed to elicit participants to define clinical integration as well as discuss key success factors of clinical integration, see below:

- What areas within the hospital require the most clinical integration?

- What has been this hospital's experience with clinical integration?

- How would you define clinical integration?

- Does clinical integration more strongly affect patient care outcomes or hospital finances, or does it affect both equally?

- What are the key functional areas and hospital management aspects to look at when defining clinical integration?

- What are some of the key success factors of clinical integration?

- If you were to measure clinical integration, what as- 
pects of the hospital would you look at?

- On a scale of 1-5 with 1 being barely clinically integrated and 5 being fully clinically integrated, how clinically integrated do you feel your hospital is?

- If you perceive your hospital as integrated, what does your hospital do to make it clinically integrated? If not, what should a hospital do to become integrated?

- Are there any hospitals in Pennsylvania that are good models of clinical integration (besides yours [if applicable])? Which ones?

- What are the major challenges to hospitals in achieving clinical integration?

\subsection{Data collection}

All interviews took place between July 12, 2013 and August 8, 2013. Each interview lasted approximately 30-minutes and was audio recorded and transcribed in its entirety. Three interviews were done in person and the remaining 10 were performed by phone.

\subsection{Data analysis}

Each interview transcript was reviewed and analyzed. Major themes were identified from each interview. The interviewer conducted, transcribed, and analyzed all interviews. Two separate individuals audited and verified the accuracy of the interview transcription and analysis. Analysis of each interview followed techniques developed by Mayring. ${ }^{[8]}$ Within each transcription, relevant text passages that related to the research question were identified and the text was reduced by paraphrasing, with any duplicate text deleted. The paraphrased text was then organized into categories of "key success factors of clinical integration". Any discrepancies were discussed and modified based on consensus. The top five most mentioned categories were included in the results and identified as key success factors for clinical integration.

\section{Results}

\subsection{Definition and perspectives of clinical integra- tion}

All participants were asked the identical question: "How would you define clinical integration?" The verbatim answer from each participant is listed.

Table 2 highlights the wide range of perceptions that exist regarding clinical integration. Thematic analysis revealed these varied definitions of clinical integration provided by health system leaders could be grouped into two categories. Eight participants (participants \#: 1, 3, 4, 5, 6, 8, 10, 13) expressed a perspective of clinical integration that focused on administrative services as important features of clinical integration. Five participants (participants \#: 2, 7, 9, 11, 12) provided a perspective of clinical integration that focused on patients or populations. Participants were also asked to score the clinical integration of their health system or network on a Likert scale of 1 (not clinically integrated) to 5 (completely clinically integrated).

Table 2: Verbatim definitions of clinical integration as provided by each participant when asked "How would you define clinical integration?"

\begin{tabular}{|c|c|c|}
\hline Participant & Definition & Self-Reported Score \\
\hline 1 & Having links between different providers & 2 \\
\hline 2 & Connection and coordination between the patient and provider & $2-3$ \\
\hline 3 & Employing doctors on the outpatient as well as the inpatient side & $4-5$ \\
\hline 4 & $\begin{array}{l}\text { Clinicians coming together, setting up standards of care, and then holding themselves } \\
\text { accountable for both improving quality and reducing cost }\end{array}$ & 5 \\
\hline 5 & Combining clinical and administrative services & 5 \\
\hline 6 & Aligned mission & 4 \\
\hline 7 & $\begin{array}{l}\text { Care coordination across the spectrum of healthcare, from primary care office to whatever } \\
\text { other part of the healthcare system patients go }\end{array}$ & $2-3$ \\
\hline 8 & Having structures in place that address the goals of quality, access and cost & 3 \\
\hline 9 & $\begin{array}{l}\text { A functioning system of interdisciplinary and intradisciplinary services to provide } \\
\text { evidence-based, or guided practice, continuous improvement and a collaborative approach } \\
\text { for seamless care of the patient }\end{array}$ & 4 \\
\hline 10 & $\begin{array}{l}\text { A system that is physician-led, professionally managed, and organized around service lines } \\
\text { with care coordination across the continuum of care }\end{array}$ & $3-4$ \\
\hline 11 & All parties working together for the betterment of the patient & 3 \\
\hline 12 & Having the resources to provide all facets that populations need to remain healthy & 2 \\
\hline 13 & Developing new, innovative ways to practice that improve outcomes and lower costs & 5 \\
\hline
\end{tabular}




\subsection{Key success factors of clinical integration}

All participants were asked "What are some of the key success factors for clinical integration?". Table 3 lists the top five success factors ranked by the number of participants who included them as well as common themes within each success factor. Each of the five success factors is discussed in more detail below.

\subsubsection{Physician alignment}

Ten of the 13 participants identified physician alignment as a key success factor for clinical integration. All participants that identified physician alignment as a key success factor agreed that in order to be truly clinically integrated, physi- cians must have a close relationship with the health system or network. Some participants $(n=3)$ identified physician alignment as direct employment of physicians within the health system. One participant stated "clinical integration really is the fact that we own the doctors and really can control the flow of patients by virtue of the fact that we own the doctors including both the inpatient and the outpatient side". Through employment, "we can be fairly specific about what the expectations are, we also hold physicians to productivity standards as well. When you're working with independent physicians, you can't do that". Similarly, another participant stated that "if you employ the physicians, it gives you an opportunity to optimize coordination of care".

Table 3: Key success factors of clinical integration identified by participants and the main themes associated with each success factor*

\begin{tabular}{|c|c|}
\hline $\begin{array}{l}\text { Key Success Factor } \\
\text { (\# of Participants) }\end{array}$ & Main Themes \\
\hline \multirow{7}{*}{ Physician Alignment (10) } & Alignment of physicians to the hospital \\
\hline & Physician leadership driving the integration \\
\hline & All physicians have an aligned mission \\
\hline & Open line of communication with the physicians \\
\hline & Employing the physicians \\
\hline & A physician-leader and administrative director for each service line \\
\hline & Employing physicians and having physician leadership \\
\hline \multirow{5}{*}{ Shared Data and Analytics (8) } & Good predictive capacity \\
\hline & Obtaining and utilizing data to better manage \\
\hline & Collecting data and using it to drive decision making \\
\hline & Heavily using data and analytics to determine outcomes of cost and quality \\
\hline & Capturing and analyzing data to understand outcomes \\
\hline \multirow{6}{*}{ Culture (7) } & Shared culture \\
\hline & A culture of physicians that is open to working with administrators \\
\hline & Team-based culture \\
\hline & Shared vision and shared goals between physicians and administrators \\
\hline & Joint decision-making between the medical staff, the clinical staff and the administrative staff \\
\hline & A physician culture willing to adapt to change \\
\hline \multirow{5}{*}{ Patient Engagement (7) } & Patient-centered medical home \\
\hline & Patient access to information about their health \\
\hline & All services that the patient needs are aligned and focused on the care of the patient \\
\hline & The use of patient care navigators \\
\hline & Team-based care with the patient at the center of that team \\
\hline \multirow{4}{*}{ Primary Care (6) } & Protocol-driven, data collecting primary care office \\
\hline & Aligned primary care office \\
\hline & Specialists communicating with primary care physicians \\
\hline & Commitment to primary care \\
\hline
\end{tabular}

Note. ${ }^{*}$ Key success factors are in order (highest to lowest) of how many participants identified each success factor.

Six participants identified physician alignment as an agreement between physicians and administrators on a cohesive ideal system regarding healthcare delivery. One participant stated "Just because you are employed, does not necessarily mean you are aligned and integrated". Another participant noted "we have several members of the physician group who are in key areas of leadership, so we have a chief physician executive, who we work closely with, and we also have within our medical group, a president of the medical group that we work closely with through our operational team". One participant stated that "our physician group meets once a month and we incentivize them (physi- 
cians) to attend those meetings... a portion of any incentive they might receive at the end of the year is partially based on attendance at a substantial number of those meetings". The participant elaborated, "what we do with those meetings is to try to further deepen that integration... we showcase some new physicians to the network, most often those are specialists, so that physicians know this is to whom they should refer for that service".

\subsubsection{Shared data and analytics}

Eight participants identified shared data and analytics as a key success factor of clinical integration. Within this category, all participants agreed that using electronic medical records to optimize shared data and analytics is a vital component to clinical integration. One executive noted "To really be clinically integrated, you really need a good information system, both electronic health record and an information system that you can look at data regarding utilization, cost, that kind of thing". Another participant stressed "we're working pretty hard to develop a shared record so that it really isn't owned by a hospital or a doctor's office, it's owned by everybody including the patient".

Five participants highlighted the importance of the use of data and analytics to develop evidenced-based protocols and to examine cost and quality. One participant, who considered their health system completely integrated (5 out of 5) noted: "we do have a data warehouse; we've collected patient data for a long time, more than a decade, where people can access the type of data they would need".

Two participants also highlighted the importance of patient access to their own electronic health record in clinical integration. One participant predicted that the future of clinical integration "will move toward putting the patient in a role of really having access to information where they can make critical decisions about their health and be more patientcentric".

\subsubsection{Culture}

Seven participants emphasized the importance of creating a culture conducive to clinical integration. One stated that the culture at their institution "is ever in evolution, but it is team-oriented, it is progressive, it expects participants to communicate well, it expects evidence-based decision making, and openness and transparency in that process". The same participant described the "acculturation" process for physicians: "we have several written documents that outline the expected behaviors between individuals in our organizations and our relationships to our patients... before a person joins our medical group, they read this and then we ask them to sign it. It's not like they're signing a legal document, it's more symbolic that these are the things we stand for and this is what we are trying to do".

One participant stressed the importance of a culture that can 40 adapt to change: "You really have to have a culture that wants to move into the future". Several participants pointed out the importance of engaging physicians to cultivate a culture of learning and change. One executive stated "you have to let physicians know, what's in it for me, what's in it for my patients, and if you can't answer those questions, don't even try to go to physicians with a new idea". One participant stated "it does mean trust, it does mean giving up autonomy, it means that I have to be willing to standardize in certain areas and I have to be willing to measure my outcomes". Another participant also mentioned the importance of a culture of trust by commenting "we went through an exercise here... listening to all of our employees... and got 10,000 ideas that were then word-sorted, and the words that fell out most frequently were, what do you need to be successful as: time, trust, and teamwork". One participant stressed joint-decision making and teamwork by stating, "I would suggest that culture, and having a culture of physicians who are open to working with one another and working with hospital administrators and health system administrators is key to the success of clinical integration... you can have the best electronic medical record in the country, and if there's not a culture of being open to implementing it, it will fall flat on its face".

\subsubsection{Patient engagement}

Seven participants identified patient engagement as a key success factor of clinical integration. One participant commented "it's really about having integration and uniformity of all the services to create a continuum for the patient beginning with outpatient care all the way through inpatient care, then out to post-acute care as well". Another participant shared similar thoughts, saying that a large part of being clinically integrated is ensuring "that there's coordination of care from the time they (patients) leave the primary care office to what other part of the healthcare system they go to". Another participant stated that in regards to clinical integration, "if you don't have engaged patients, that's a recipe for failure".

Two participants mentioned the role of patient-care navigators in clinical integration and several others highlighted specific programs that have been enacted to increase clinical integration through patient engagement. One participant said "we've involved behavioral health specialists to help with those patients that have chronic disease or that are high utilizers of service", and "we have what we call care coordination teams that we're assigning to some of these practices out in the community and identifying those patients that need greater attention". In addition, health systems are looking for creative ways to incorporate patient inputs. One participant described: "We've had retreats and we invite patients, and we often invite patients that have had bad experiences that have written to us, and put them in front of a hundred of our manager-level people to tell their story of 
what they experience".

Three participants mentioned the importance of patient access to their own health records, referred to as a "patient portal". One participant highlighted the importance of population health: "We're working on certain common health conditions, the management of pneumonia for example, or everybody has to worry about diabetes these days, or obesity and we have task forces working on better outcomes in helping manage people who have those conditions".

\subsubsection{Primary care}

Six participants identified primary care as a key success factor of clinical integration. One participant stated that the primary care office is "the portion of any health system that the patient identifies as their contact point", and it is crucial that the primary care office is integrated with the rest of the health system.

Four participants mentioned the Patient-Centered Medical Home model as a key component of clinically integrated health systems. One participant stated "that's really a building block of clinical integration, is a medical home for patients, especially for the chronic disease patients". Another executive stressed that "I think in the future hospitals are going to be nothing but cost centers, and really where all the action is going to be is really the outpatients. It's going to be in the primary care centers, it's going to be in that medical home, and if you're able to coordinate care appropriately with care management, disease management, you're really going to be able to keep patients out of the hospital and so that's really why I say that hospitals within a clinically integrated healthcare model are really going to be a cost center".

One participant commented that "the key to looking at the new environment starts with primary care and are they protocol driven, and are they collecting the data and returning it to the physician so that they can measure the effectiveness of their own care". The alignment and integration of not only the primary care physicians, but the office as a whole is a key success factor to clinical integration programs.

\section{Discussion}

Clinical integration means different things to different health systems. As demonstrated in this study, even the characteristics of a health system have an effect on how clinical integration is perceived. Although there is no clear consensus about what clinical integration entails, two main viewpoints were identified from the interviews: providercentric clinical integration and patient-centric clinical integration.

Eight participants expressed a provider-centric perspective of clinical integration which focused on leadership roles, organizational structure, and physician alignment. Other provider-centric themes were frequently mentioned: em- ploying the physicians, holding physicians accountable, alignment of mission, physician leadership, having appropriate structure in place and improving quality and lowering costs. These themes focused on strengthening leadership and structure. Interestingly, one participant used the wording "ownership" to reflect physician employment. This may indicate that some hospital administrators view employment as a means to align physicians with the vision of the hospital or health system by which they are employed.

Five participants provided a patient-centric perspective of clinical integration. They frequently used terms such as "patient" or "population". Other patient-centric themes included: connecting patients to their providers, coordinated and seamless care of the patient, working together for the patient, and providing resources to keep populations healthy. These participants specifically emphasized the importance of the patient's experience and strengthening patient involvement in their own healthcare.

Participants were asked to score the clinical integration of their health system or network on a Likert scale of 1 (not clinically integrated) to 5 (completely clinically integrated) (see Table 2). Participants with provider-centric definitions reported scores of 2-5, with four of eight reporting 5. Participants with patient-centric definitions reported scores of 2-4. Overall, participants who see clinical integration from the provider-centric perspective perceive their health system or network as more clinically integrated than those of the patient-centric perspective.

Furthermore, participants' viewpoints on clinical integration closely reflect the basic characteristics of the health system's flagship hospital. Participants from larger hospitals (range: 469-1,763 beds; median number of employee: 4,754 ) were more likely to express provider-centric themes, whereas those from smaller hospitals (range: 134-623 beds; median number of employee: 1,774) more often discussed patient-centric themes. Therefore, the provider-centric view of clinical integration was associated with participants from larger institutions whereas the patient-centric view of clinical integration was associated with participants from smaller institutions. A limitation to this conclusion is that all but one of the participants was from an urban hospital. Further studies including more rural hospitals could provide more comprehensive results and yield new information.

Although there is no clear consensus on the definition of clinical integration, key success factors were identified in this study, namely: physician alignment, shared data and analytics, culture, patient engagement, and primary care. There are many quality initiatives evolving around clinical integration without an explicit and shared understanding of what clinical integration means. Although the "scorecard" created by Devers et al. still holds value in today's healthcare environment, the concept of clinical integration is becoming more complex. 


\section{Conclusion}

Although two main perspectives are identified, providercentric and patient-centric, a fundamental question remains unanswered: how to define clinical integration? Regardless of a provider-centric or patient-centric perspective, there should be a common definition of clinical integration addressing both. These two perspectives are not mutually exclusive; rather, both must be employed to truly represent clinical integration. Based on the results of this study, an updated definition of clinical integration must include: using features in organization, employment and alignment to improve the patient-centered experience, and using connection, coordination, and empowerment of the patient to guide the objectives for alignment and organization of a health system.

A recent study found that different health care characteristics associated with Accountable Care Organizations, and other integrated healthcare systems, were associated with different cost and quality outcomes. Their findings indi- cated that healthcare systems that became more centralized over the study period showed larger reductions in mortality, but developing specific types of physician alignment was associated with increased mortality. ${ }^{[9]}$ This may be cause for concern, as physician alignment was the most mentioned "key success factor for clinical integration" named by the health system leaders who participated in this study.

The objective of this study was to help start the conversation aimed at clarifying the concepts comprising clinical integration programs. While it was outside the scope of this study, it will be important moving forward to determine what impact - negative or positive - clinical integration initiatives have on healthcare systems and patient outcomes. Larger scale interviews and empirical studies are necessary to develop a comprehensive understanding of clinical integration.

\section{Acknowledgements}

The authors wish to thank the health system executives who participated in this study for their time and insight.

\section{References}

[1] Berwick DM, Nolan TW, Whittington J. The triple aim: care, health, and cost. Health Aff (Millwood). 2008; 27(3): 759-69. PMid: 18474969. http://dx.doi.org/10.1377/hlthaff.27.3.759

[2] Gillies RR, Shortell SM, Anderson DA, et al. Conceptualizing and measuring integration: findings from the health systems integration study. Hospital \& health services administration. 1993; 38(4): 467 89. PMid: 10130608.

[3] Hwang W, Chang J, Laclair M, et al. Effects of integrated delivery system on cost and quality. The American journal of managed care. 2013; 19(5): e175-84. PMid: 23781916.

[4] Devers KJ, Shortell SM, Gillies RR, et al. Implementing organized delivery systems: an integration scorecard. Health care management review. 1994; 19(3): 7-20. PMid: 7822193.

[5] Dubbs NL, Bazzoli GJ, Shortell SM, et al. Reexamining organizational configurations: an update, validation, and expansion of the taxonomy of health networks and systems. Health services research. 2004; 39(1): 207-20. PMid: 14965084. http://dx.doi .org/10. 1111/j.1475-6773.2004.00222.x

[6] Health Policy Brief: Medicare Readmissions Reduction Program. Health Affairs [Internet]. November 12, 2013. Available from: http://www.healthaffairs.org/healthpolicybrie fs/brief .php?brief_id=102

[7] Berkwits M, Inui TS. Making use of qualitative research techniques. Journal of general internal medicine. 1998; 13(3): 195-9. PMid: 9541377.

[8] Mayring P. Qualitative Content Analysis. A Companion to Qualitative Research. 2000.

[9] Chukmaitov A, Harless DW, Bazzoli GJ, et al. Delivery system characteristics and their association with quality and costs of care: Implications for accountable care organizations. Health care management review. 2014. PMid: 24566250. http://dx.doi .org/10.1097/H MR. 0000000000000014 